\title{
INDUTORES E EFEITOS DO DESENVOLVIMENTO DO ENOTURISMO NAS VINIICOLAS: A PERSPECTIVA DAS CAPACIDADES DINÂMICAS ${ }^{1}$
}

\author{
DRIVERS AND EFFECTS OF WINE TOURISM DEVELOPMENT IN WINERIES: THE PERSPECTIVE OF \\ DYNAMIC CAPABILITIES
}

\section{INDUCTORES Y EFECTOS DEL DESARROLLO DEL ENOTURISMO EN BODEGAS: LA PERSPECTIVA DE LAS CAPACIDADES DINÁMICAS}

Joice Lavandoski

Docente da Universidade Federal do Estado do Rio de Janeiro (Unirio) Doutora em Turismo, Faculdade de Economia, Universidade do Algarve (Ualg) Membro CIEO (Ualg) joicelavandoski@yahoo.com.br

João Albino Silva

Docente da Faculdade de Economia, Universidade do Algarve (Ualg) Doutor em Economia, Universidade Técnica de Lisboa (UTL) Membro CIEO (Ualg) jsilva@ualg.pt

\author{
Alfonso Vargas-Sánchez \\ Docente da Universidade de Huelva (UHU) \\ Doutor em CC. Econômicas e Empresariais, Universidade de Sevilha (US) \\ Coordenador GEIDETUR (UHU) \\ vargas@uhu.es
}

Patrícia Susana Lopes Guerrilha dos Santos Pinto Docente da Faculdade de Economia, Universidade do Algarve (Ualg) Doutora em Métodos Quantitativos aplicados à Economia e à Gestão, Universidade do Algarve (Ualg) Vice-presidente CIEO (Ualg) pvalle@ualg.pt

Data de Submissão: 02/08/2016

Data de Aceitação: 10/10/2016

RESUMO: No contexto organizacional das vinícolas, o enoturismo envolve a entrada dessas empresas no setor terciário da economia, por meio de um conjunto de serviços e atividades turísticas. Este estudo baseia-se na abordagem de capacidades dinâmicas ao propor que as vinícolas propositadamente criam, ampliam e modificam os seus processos, construindo e utilizando capacidades dinâmicas-chave para desenvolver o enoturismo na sua unidade de negócio. Formularam-se dois objetivos para este estudo. O primeiro objetivo é compreender em que medida os níveis de capacidades dinâmicas contribuem para o desenvolvimento do enoturismo nas vinícolas. O segundo objetivo é analisar os efeitos do desenvolvimento do enoturismo no desempenho organizacional. A abordagem Partial Least Squares (PLS), - Modelo de Equações Estruturais (SEM) e os dados de uma pesquisa quantitativa são aplicados nas vinícolas da região do Alentejo, Portugal. Este estudo mostra os indutores das capacidades dinâmicas sobre o comportamento das vinícolas para desenvolver o enoturismo e o efeito do enoturismo no desempenho organizacional. O resultado mostra que as vinícolas renovam e alargam as suas capacidades operacionais. Um conjunto de novas capacidades-chave (detectar, aprender, integrar, coordenar e reconfigurar) contribuem simultaneamente, e de forma diferente, para o desenvolvimento do enoturismo. Além disso, o desenvolvimento do enoturismo tem impacto positivo no desempenho das vinícolas.

1 Este trabalho foi possível realizar graças a agência de fomento à pesquisa fornecido pela Capes - Coordenação de Aperfeiçoamento de Pessoal de Nível Superior,_através da bolsa de estudo de doutorado pleno no exterior (processo $n^{\circ} 5618 / 10-0$ ), e pelo financiamento proveniente da FCT Fundação para a Ciência e Tecnologia (projeto UID/SOC/04020/2013). 
PALAVRAS-CHAVE: Desenvolvimento do enoturismo. Capacidades dinâmicas. Mudança estratégica.

ABSTRACT: In the organizational context of the wineries, wine tourism involves the winery's entry into the tertiary sector of the economy through a set of tourism services and activities. This study draws on the dynamic capabilities approach to propose that wineries purposely create, extend, and modify their processes by building and using key dynamic capabilities to develop wine tourism in their business portfolios. Two objectives were formulated for this study. The first was to understand the extent to which the businesses' levels of dynamic capabilities can contribute to the development of wine tourism in wineries. The second was to analyze the effects of wine tourism development on the wineries' organizational performance. The partial least squares (PLS) method, structural equation modeling (SEM), and data from a quantitative survey were applied to the business Alentejo wineries, in Portugal. This study shows the drivers of dynamic capabilities on the wineries' behavior towards wine tourism development, and the effect of wine tourism on wineries' organizational performance. The result shows that wineries are renewing and extending their operational capabilities. A set of new key capabilities (sensing, learning, integrating, coordinating, and reconfiguring) contribute simultaneously and differentially to the development of wine tourism. This development, in turn, is positively impacting the wineries' performance.

KEYWORDS: Wine tourism development. Dynamic capabilities. Strategic change.

RESUMEN: En el contexto organizacional de las bodegas, el enoturismo implica la entrada de estas empresas en el sector terciario de la economía a través de un conjunto de servicios y actividades turísticas. Este estudio se basa en el enfoque de las capacidades dinámicas, proponiendo que las bodegas intencionalmente crean, amplían y modifican sus procesos mediante la construcción y el uso de las capacidades dinámicas clave para el desarrollo del enoturismo en su unidad de negocio. Se formularon dos objetivos para este estudio. El primer objetivo es comprender en qué medida los niveles de capacidades dinámicas contribuyen al desarrollo del enoturismo en bodegas. El segundo objetivo es analizar los efectos del desarrollo del enoturismo en el desempeño organizacional. El enfoque Partial Least Squares (PLS), el Modelo de Ecuaciones Estructurales (SEM) y los datos de la investigación cuantitativa se aplican a las bodegas de la región del Alentejo, Portugal. Este estudio muestra los inductores de las capacidades dinámicas sobre el comportamiento de las bodegas para desarrollar el enoturismo y el efecto del enoturismo en el desempeño organizacional. El resultado muestra que las bodegas renuevan y amplían sus capacidades operativas. Un conjunto de nuevas capacidades clave (detectar, aprender, integrar, coordinar y reconfigurar) contribuyen de forma simultánea y diferenciada en el desarrollo del enoturismo. Además, el desarrollo del enoturismo tiene un impacto positivo en el desempeño de las bodegas.

PALABRAS CLAVE: Desarrollo del enoturismo. Capacidades dinámicas. Cambio estratégico.

INTRODUÇÃO existência do enoturismo depende, em grande medida, da
combinação e de um relacionamento harmonioso entreas indústrias
do vinho e do turismo (Alonso \& Liu, 2012; Iglesias \& Navarro, 2014; Telfer, 2001). Estudos identificam alguns problemas nesta relação, tais como: (a) as vinícolas não estão focadas no produto enoturístico; (b) há escassez de informações sobre turismo na indústria vinícola; (c) há falta ou uma fraca integração entre os produtores de vinho e; (d) pouca cooperação interindústrias (Alonso \& Liu, 2012; Beames, 2003; Dowling \& Carlsen, 1998; Macionis, 1997).

Para a indústria vinícola, o enoturismo é uma expansão do negócio vitivinícola; uma oportunidade de diversificação do negócio. Também é uma forma de agregar valor ao principal produto da empresa, o vinho (Iglesias \& Navarro, 
2014). Estudos destacam benefícios para as vinícolas com o enoturismo, tais como: aumento dos lucros e das vendas, melhorias na reputação da marca e na qualidade dos produtos, favorecendo assim a competitividade organizacional. O enoturismo também pode ajudar a educar os clientes e os consumidores para o vinho (Beverland \& Lockshin, 2001; Carlsen, 2004; Christou \& Nella, 2010; Dowling \& Carlsen, 1998; Stavrinoudis, Tsartas, \& Chatzidakis, 2012). Existem vinícolas que reconhecem o enoturismo como uma possibilidade de sobrevivência para o seu negócio (Hojman \& Hunter-Jones, 2012), ainda que esta atividade/segmento tenha importância secundária ou terciária na empresa (Iglesias \& Navarro, 2014). Em contrapartida, há vinícolas alcançando benefícios diretos muito limitados, ou nenhum benefício, por meio do seu envolvimento com o enoturismo (Alonso \& Liu, 2012).

No contexto organizacional, o enoturismo requer o ingresso das vinícolas no setor terciário da economia, oferecendo um conjunto de serviços e atividades turísticas em paralelo com as atividades principais da empresa, as quais estão relacionadas com a agricultura e a produção de vinho. No entanto, o ambiente produtivo da indústria vinícola e da indústria do turismo está em lado oposto ao escopo industrial (Carlsen, 2004) e existem diferentes condições econômicas aplicáveis ao vinho e ao turismo. Estas características são essencialmente diferentes em um sentido microeconômico, em termos de oferta, demanda, produto/serviço, renda/lucro, crescimento, qualidade, dentre outros (Carlsen, 2004). Estes aspectos podem explicar por que as vinícolas, em geral, têm uma forte orientação para o produto, neste caso, a produção de vinho, e possuem pouco conhecimento sobre o turismo (Dowling \& Carlsen, 1998; Iglesias \& Navarro, 2014; Macionis, 1997).

Questões sobre a natureza de desenvolvimento do enoturismo ainda necessitam de esclarecimentos (Carlsen, 2004; Dowling \& Carlsen, 1998; Getz, 2000; Hall, Sharples, Camborne, \& Macionis, 2002). A literatura ainda não conseguiu explicar como as vinícolas se adaptam para o enoturismo. Este estudo pretende contribuir nesta área, propondo que o envolvimento das vinícolas com o setor de serviços, por meio da agregação da componente de enoturismo no ramo de atividade empresarial (como uma expansão do negócio vitivinícola), origina um processo inovador que guia as empresas a este tipo de atividade 
ISSN: 1983-7151

que envolve serviços turísticos. Neste sentido, elas precisam mudar processos internos e aprender a desenvolver atividades, até então, não rotineiras. Ou seja, agregar o enoturismo implica novas rotinas, processos e capacidades organizacionais. Pois, em certa medida, as vinícolas necessitam ajustar/mudar o seu modelo de negócio para incorporar o enoturismo.

A presente investigação pretende mostrar que o enoturismo provoca uma série de mudanças nos processos, nas estruturas organizacionais, na tomada de decisão e na capacidade estratégica das organizações para atender às necessidades dos enoturistas. Alguns exemplos são a mobilização dos recursos endógenos para a criação de unidades de alojamento a partir da infraestrutura existente (edifícios históricos e tradicionais), ou a utilização e a adaptação de espaços que, até então, são utilizados de forma exclusiva para a atividade agrícola, e que passam a ser também usados para o turismo. Neste sentido, o enoturismo envolve um processo de mudança estratégica que pode impactar e mudar o modelo de negócio dessas empresas, permitindo que elas combinem produção e manufatura (produção de vinho orientada para a agricultura, na indústria vinícola), com visitas e degustações na adega (prestação de serviços orientada para o turismo, na indústria do turismo), que fornecem uma experiência vínica aos visitantes (Carlsen, 2004; Iglesias \& Navarro, 2014; Zamora \& Barril, 2007).

Por meio da abordagem de capacidades dinâmicas, o primeiro objetivo deste estudo é compreender em que medida os seus níveis de capacidades dinâmicas contribuem para o desenvolvimento do enoturismo nas vinícolas. $O$ segundo objetivo é analisar os efeitos do desenvolvimento do enoturismo no desempenho organizacional. O estudo é baseado em um modelo conceitual estimado e validado por meio da Modelação de Equações Estruturais (SEM), e aplicado às vinícolas que possuem o enoturismo e que pertencem à Rota dos Vinhos do Alentejo, em Portugal. Pretende-se, portanto, fornecer insights no campo da gestão estratégica, por meio de uma análise do modelo de negócio intraorganizacional, que permite mensurar os indutores e os efeitos do desenvolvimento do enoturismo nas vinícolas.

As capacidades dinâmicas têm se tornado um quadro teórico para compreender as mudanças estratégicas organizacionais (Teece, Pisano \& 
Shuen, 1997). O principal argumento da abordagem de capacidades dinâmicas estabelece que a empresa necessita detectar, apreender e reconfigurar capacidades para adquirir vantagens competitivas. Estudos de referência que aplicam o conceito de capacidades dinâmicas são recentes. No entanto, em pouco tempo, tem-se verificado um desenvolvimento teórico e empírico nas diferentes áreas e setores da economia (Ambrosini, Bowman \& Collier, 2009; Ambrosini \& Bowman, 2009; Barreto, 2010; Eisenhardt \& Martin, 2000; Helfat et al., 2007; Teece, 2007, 2009; Teece et al., 1997; Winter, 2003; Zahra, Sapienza \& Davidsson, 2006; Zollo \& Winter, 2002). Alguns estudos foram aplicados no setor de serviços, particularmente no turismo (Haugland, Ness, Grønseth, \& Aarstad, 2011; Kim \& Boo, 2010; Lemmetyinen \& Go, 2009; Nieves \& Haller, 2014). Contudo, não existem estudos com esta abordagem aplicados ao desenvolvimento do enoturismo (Lavandoski, Vargas-Sánchez, \& Silva, 2014).

Este artigo está organizado da seguinte forma: a próxima seção revisa a literatura sobre enoturismo e capacidades dinâmicas, apresenta estudos que examinam os processos de desenvolvimento das capacidades dinâmicas e os seus efeitos no setor de serviços, particularmente em empresas turísticas. Posteriormente, são propostos o modelo conceitual e as hipóteses de investigação. A metodologia apresenta os métodos e os dados utilizados no estudo. Em seguida, discutem-se os principais resultados da análise. A seção final, além de fornecer conclusões e implicações teóricas e práticas, também reflete sobre as limitações e oferece algumas recomendações para futuras pesquisas.

\section{FUNDAMENTAÇÃO TEÓRICA}

\section{ENOTURISMO}

O conceito de enoturismo surge da ligação do produto vinho com a atividade turística. A Carta Europeia do Enoturismo, como o documento mestre que estabelece os fundamentos e os princípios do enoturismo na Europa, define enoturismo como um conjunto de todas as atividades e recursos turísticos, de 
lazer e de tempo livre, relacionados com as culturas materiais e imateriais do vinho e da gastronomia autóctone.

Nos diferentes países produtores de vinho, o desenvolvimento do enoturismo se baseia, sobretudo, na criação e na estruturação de rotas ou estradas oficiais de vinhos, geridas por uma entidade, geralmente de caráter associativo (Hall et al., 2002). Paralelamente às rotas de vinho, é preciso que as empresas vinícolas desenvolvam o enoturismo na sua unidade de negócio, com infraestrutura para receber os visitantes nas suas instalações, programas de visitação e atividades que envolvam o vinho (como passeios nos parreirais, degustação de vinhos, atividades de vindima, dentre outras).

A Europa é um grande destino emissor e receptor de enoturistas e é onde o fluxo de visitantes nas vinícolas aumenta expressivamente. Em Portugal, a atividade enoturística já se encontra bastante difundida, muito em função da forte tradição cultural dos portugueses com o vinho e da crescente quantidade de empresas vinícolas investindo no enoturismo. Além disso, o enoturismo é um produto estratégico para o desenvolvimento do turismo em Portugal. Dentro do escopo "gastronomia e vinho", o enoturismo é um dos dez pilares que sustentam a promoção turística do país, conforme estabelecido no Plano Estratégico Nacional de Turismo - PENT 2013-2015 (Turismo de Portugal, 2013). O país possui 11 rotas de vinhos, distribuídas por 12 regiões de produção vitivinícolas (Instituto da Vinha e do Vinho, 2015), que começaram a ser implementadas a partir de 1993, por meio do programa comunitário "Dyonisios" da União Europeia.

\section{CAPACIDADES DINÂMICAS}

Este estudo baseia-se na abordagem de capacidades dinâmicas ao argumentar que as vinícolas propositadamente criam, ampliam e modificam os seus processos, construindo e utilizando capacidades dinâmicas-chave para desenvolver o enoturismo na sua unidade de negócio. Neste contexto organizacional, estas capacidades dinâmicas permitem analisar as mudanças nos processos estratégicos que conduzem ao desenvolvimento do enoturismo e o seu efeito no desempenho organizacional. 
Capacidades dinâmicas é uma abordagem emergente da gestão estratégica com uma preocupação centrada na reconfiguração de recursos e de capacidades organizacionais. Tal abordagem começou a ser estudada após a Teoria da Visão Baseada em Recursos (RBV) ter sido considerada inadequada para explicar a vantagem competitiva sustentável das organizações em ambientes de rápida mudança (Teece, 2009; Teece \& Pisano, 1994). As capacidades dinâmicas foram inicialmente definidas como "a capacidade da empresa para integrar, construir e reconfigurar competências internas e externas, para lidar em ambientes de rápida mudança" (Teece, Pisano \& Shuen, 1997, p. 516). A partir deste estudo seminal, um amplo corpo de pesquisadores tem avançado, primeiramente com quadros teóricos e, posteriormente, com estudos empíricos nas diversas áreas de pesquisa. Segundo Winter (2003) e Teece (2007), Pavlou e El Sawy (2011, p. 242) entendem "capacidades dinâmicas como aquelas capacidades que ajudam a ampliar, modificar, e reconfigurar as suas capacidades operacionais existentes em novas, que melhor combinam com o ambiente em mudança", em que "capacidades operacionais" são "definidas como a capacidade/habilidade de executar atividades do dia a dia".

As capacidades dinâmicas diferem de outras habilidades organizacionais, de acordo com a importância e a natureza que elas assumem na organização (Barreto, 2010). Elas estão localizadas em um segundo nível hierárquico (Winter, 2003). Capacidades dinâmicas têm uma natureza transformacional relacionada com ciclos de mudança organizacional e operam para, propositadamente, criar, ampliar ou modificar a base de recursos da empresa localizada no primeiro nível hierárquico (Helfat et al., 2007). Há um consenso de que as capacidades dinâmicas são um tipo específico de capacidade organizacional que é única e específica para uma organização (Eisenhardt \& Martin, 2000; Teece, 2009; Teece et al., 1997). Assim, em um conjunto de organizações, existem capacidades dinâmicas heterogêneas (Teece et al., 1997; Zahra et al., 2006). Capacidades dinâmicas não podem ser compradas; elas devem ser construídas (Prahalad \& Hamel, 1990; Teece et al., 1997; Teece \& Pisano, 1994) e são difíceis de replicar ou imitar (Teece, 2009; Teece \& Pisano, 1994). Capacidades dinâmicas geralmente ocorrem apenas de forma incremental (Teece \& Pisano, 1994). Eisenhardt e Martin (2000) reconhecem o potencial das capacidades dinâmicas 
como uma ferramenta para manipular a configuração de recursos em busca de uma eficácia melhorada.

Um conjunto específico de processos subjacentes explicam como as capacidades dinâmicas funcionam. O estudo original de Teece et al. (1997) apresenta os microfundamentos das capacidades dinâmicas, que são: coordenar/integrar, aprender e reconfigurar. Posteriormente, Teece (2007) reagrupa os microfundamentos em três fases: (a) detectar, (b) apreender, e (c) gerir ameaças e reconfigurar. A primeira fase refere-se à identificação e à avaliação de oportunidades no ambiente. A segunda fase do processo consiste em mobilizar os recursos necessários para as oportunidades identificadas, definindo estratégias e gerando valor por meio destas operações. A terceira fase, gerir ameaças e reconfigurar, é a renovação contínua dos recursos e das rotinas organizacionais necessárias para manter uma vantagem competitiva. Considerando os argumentos de Teece, estudos empíricos recentes propõem um modelo mensurável para representar a natureza das capacidades dinâmicas (Pavlou \& El Sawy, 2011, 2013; Protogerou, Caloghirou, \& Lioukas, 2012). Estes estudos estabelecem que as capacidades dinâmicas têm um efeito positivo indireto no desempenho através da reconfiguração de capacidades operacionais. Segundo o ponto de vista de Protogerou et al. (2012), as capacidades dinâmicas envolvem a coordenação, a aprendizagem e a resposta competitiva estratégica. Diferentemente, Pavlou e El Sawy (2011, 2013) apresentam quatro componentes das capacidades dinâmicas: detectar, aprender, integrar e coordenar.

A capacidade de detectar refere-se à habilidade de identificar, interpretar e buscar oportunidades no ambiente. A capacidade de aprender é a habilidade de renovar as capacidades operacionais existentes com novos conhecimentos. Capacidade de integrar envolve a habilidade de combinar o conhecimento individual em novas capacidades operacionais na empresa. Finalmente, a capacidade de coordenar refere-se à habilidade de orquestrar e implantar tarefas, recursos e atividades nas novas capacidades operacionais. Estas quatro capacidades dinâmicas interagem em uma lógica sequencial que permite reconfigurar as capacidades operacionais existentes (Pavlou \& El Sawy, 2011, 2013). 
Teece (2009) reconhece que as capacidades dinâmicas e a inovação estão conectadas. Esta relação pode ser identificada na seguinte afirmação: "capacidades dinâmicas com certeza requerem a criação, integração e comercialização de um fluxo contínuo de inovação consistente com as necessidades dos clientes e as oportunidades tecnológicas" (Teece, 2009, p. 52). Em relação à reestruturação e à diversificação das empresas agrícolas, o estudo de Grande (2011) revela que sem a habilidade de detectar e aproveitar as oportunidades, as empresas agrícolas provavelmente não seriam nem dinâmicas, nem adaptativas. Vários estudos aplicando a abordagem de capacidades dinâmicas no setor de serviços analisam a relação entre as capacidades dinâmicas e a inovação (Ellonen, Wikström \& Jantunen, 2009; Gebauer, 2011; Hertog, Aa \& Jong, 2010; Kindström, Kowalkowski, \& Sandberg, 2013; Salunke, Weerawardena, \& McCollKennedy, 2011).

No setor de serviços, por meio da identificação dos microfundamentos essenciais instituídos por Teece (2007), pesquisadores investigam como as capacidades dinâmicas moldam a forma como os negócios ligados ao serviço são desenvolvidos em diversas empresas de manufatura. Os principais desafios para as empresas que mudam seu modelo de negócio para desenvolver serviços envolvem gerir e expandir as capacidades dinâmicas de detectar, aprender e reconfigurar, as quais facilitam o foco no ramo de negócio ligado ao serviço e são essenciais para a inovação de serviço bem-sucedida (Fischer, Gebauer, Gregory, Ren, \& Fleisch, 2010; Gebauer, 2011; Kindström et al., 2013). Salunke et al. (2011) sugerem que as empresas de serviço com comportamento empreendedor (com proatividade e inovação, por exemplo) buscam a inovação cuidadosamente, selecionando e utilizando capacidades dinâmicas que as permitam alcançar uma maior inovação e vantagens competitivas. Assim, as capacidades dinâmicas permitem que as empresas criem novas combinações de recursos baseadas no conhecimento, levando à inovação de serviço.

A literatura sobre o desenvolvimento de novos serviços e sobre inovação de serviço mostra que os processos de desenvolvimento de serviço são diferentes dos processos de desenvolvimento de produtos (Salunke et al., 2011). As características complexas dos serviços (isto é, intangibilidade, inseparabilidade, variabilidade, perecibilidade e heterogeneidade) devem ser consideradas e, por sua vez, refletem as condições únicas da indústria de serviços. Isto pode ser 
verificado no comportamento das vinícolas para o enoturismo (objeto desta análise). O desenvolvimento do enoturismo pode ser entendido como um processo inovador nestas empresas quando elas são guiadas para desenvolver serviços turísticos.

Como identificado por Lavandoski et al. (2014), é recente e crescente a aplicação da abordagem de capacidades dinâmicas no setor do turismo. Estes estudos turísticos fornecem insights sobre os processos de desenvolvimento de capacidades dinâmicas em hotéis (Nieves \& Haller, 2014), pelos organizadores de eventos (Kim \& Boo, 2010), ou coordenadores (Lemmetyinen \& Go, 2009), bem como em destino turístico, a partir do qual emerge o conceito de capacidade de destino (Haugland et al., 2011). Nieves e Haller (2014) verificam que um maior nível de capital humano (neste caso, nível de conhecimento dos colaboradores, competências e habilidades) incentiva o desenvolvimento de capacidades dinâmicas de detectar, aprender, integrar e coordenar em hotéis espanhóis e portugueses. No contexto dos organizadores de eventos, a gestão do conhecimento e a habilidade de reconfigurar e integrar os recursos existentes em novas formas (reconfigurabilidade de recursos) são duas habilidades-chave, que impactam diretamente no desempenho dos organizadores de eventos (Kim \& Boo, 2010). Além disso, Lemmetyinen e Go (2009) identificam que as três capacidades essenciais, necessárias para gerir redes de negócios turísticos, envolvem a capacidade de parceria, a habilidade de criar conhecimento e a capacidade de absorção.

\section{MODELO CONCEITUAL E HIPÓTESES DE INVESTIGAÇÃO}

Esta revisão da literatura procurou demonstrar que os processos organizacionais estão se tornando uma maneira aceitável e promissora para analisaras capacidades dinâmicas. Estudos anteriormente apresentados mostram como as organizações mudam os processos internos por meio de capacidades dinâmicas. Estes processos incluem métodos para o desenvolvimento de novos produtos, processos de resolução de problemas, processos de partilha de conhecimento, desenvolvimento de conhecimentos em marketing, entre outros (Easterby-Smith, Lyles, \& Peteraf, 2009; Teece, 2007, 2009). Diante desta análise, 
o primeiro objetivo deste estudo é compreender em que medida os níveis de capacidades dinâmicas contribuem para o desenvolvimento do enoturismo nas vinícolas. Este artigo argumenta que as vinícolas propositadamente criam, ampliam e modificam os seus processos, construindo e utilizando capacidades dinâmicas-chave para o desenvolvimento do enoturismo. Esta reflexão conduz a seguinte hipótese:

H1: Existe uma relação positiva e direta entre as capacidades dinâmicas e o desenvolvimento do enoturismo em contexto organizacional.

Usando os componentes da capacidade de detectar, aprender, integrar e coordenar para mensurar as capacidades dinâmicas (Pavlou \& El Sawy, 2011, 2013), pesquisadores podem relacionar os resultados da investigação empírica com a literatura sobre capacidades dinâmicas de uma forma rigorosa (BarralesMolina, Martínez-López, \& Gázquez-Abad, 2014). Com base neste argumento, são propostas as seguintes hipóteses:

H1a: A capacidade de detectar, através da sua contribuição para formar as capacidades dinâmicas, influencia positivamente o desenvolvimento do enoturismo.

H1b: A capacidade de aprender, através da sua contribuição para formar as capacidades dinâmicas, influencia positivamente o desenvolvimento do enoturismo.

H1c: A capacidade de integrar, através da sua contribuição para formar as capacidades dinâmicas, influencia positivamente o desenvolvimento do enoturismo.

H1d: A capacidade de coordenar, através da sua contribuição para formar as capacidades dinâmicas, influencia positivamente o desenvolvimento do enoturismo.

H1e: A capacidade de reconfigurar, através da sua contribuição para formar as capacidades dinâmicas, influencia positivamente o desenvolvimento do enoturismo.

A utilização das capacidades dinâmicas permite que as organizações utilizem medidas estratégicas para evitar impactos negativos na organização. Eisenhardt e Martin (2000) argumentam que o valor das capacidades dinâmicas para a vantagem competitiva reside na sua habilidade de alterar a base de recursos. Em geral, os estudos assumem a existência de uma potencial influência 
positiva das capacidades dinâmicas no desempenho organizacional (Desai, Sahu, \& Sinha, 2007; García-Morales, Jiménez-Barrionuevo, \& GutiérrezGutiérrez, 2012; Grande, 2011; Hsu \& Wang, 2012; Hung, Chung, \& Lien, 2007; Hung, Yang, Lien, McLean, \& Kuo, 2010; Kim \& Boo, 2010; Leonidou, Leonidou, Fotiadis, \& Zeriti, 2013; Pavlou \& El Sawy, 2011, 2013; Protogerou et al., 2012; Sainaghi \& De Carlo, 2012; Wilden, Gudergan, Nielsen, \& Lings, 2013; Yalcinkaya, Calantone, \& Griffith, 2007). Neste estudo, pretende-se analisar o efeito do desenvolvimento do enoturismo no desempenho organizacional, afirmando que:

H2: O desenvolvimento do enoturismo influencia direta e positivamente o desempenho organizacional.

Com base na literatura apresentada, a Figura 1 apresenta o modelo de análise que, indiretamente, conecta as cinco capacidades (CDE, CA, CI, CC e CR) por meio das capacidades dinâmicas (CD), com o desenvolvimento do enoturismo (DE) e os seus efeitos no desempenho organizacional (DO). Este modelo possui um total de 103 indicadores (ou variáveis observáveis) e 8 constructos (ou variáveis latentes). As hipóteses estão representadas nos caminhos correspondentes. As linhas tracejadas representam as hipóteses que envolvem relações indiretas entre os construtos.

Figura 1: Modelo de pesquisa proposto

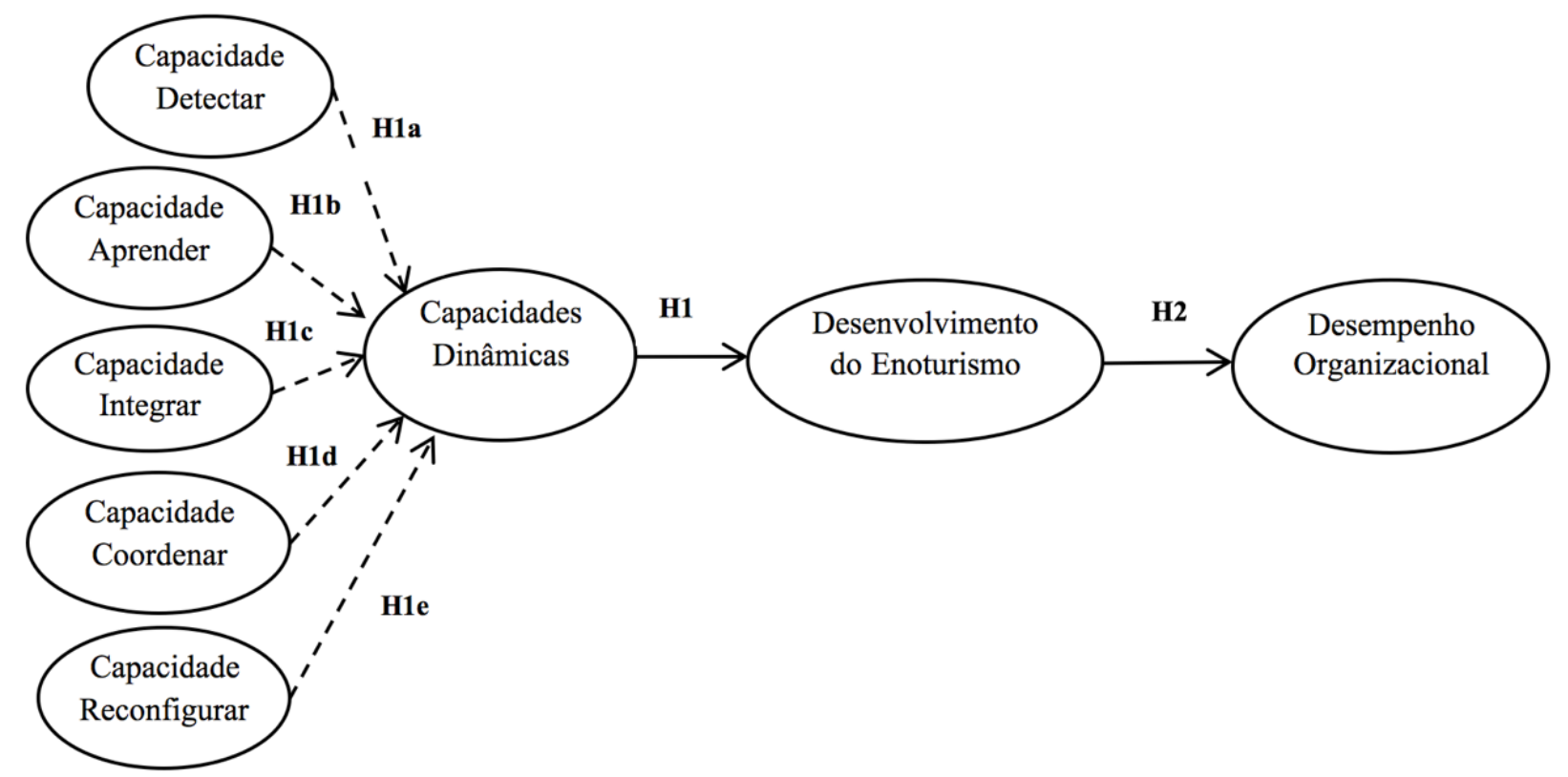

Fonte: Elaboração própria. 
A pesquisa foi realizada no Alentejo, uma das principais regiões de enoturismo localizada ao sul de Portugal, e foi escolhida devido à diversidade da oferta enoturística e às características das empresas com unidades de enoturismo em seu negócio. A região do Alentejo possui mais de 21 mil hectares de área total de vinhedos e é a maior produtora de vinhos em termos de volume, com indicação geográfica protegida (IGP) no país (Instituto da Vinha e do Vinho, 2015). A Rota dos Vinhos do Alentejo congrega 263 produtores de vinho e 97 varejistas, onde pequenas a grandes empresas se destacam no cenário nacional, em termos de produção de vinho e ampla propriedade de área agrícola com vinhedos.

Um questionário quantitativo foi elaborado e aplicado nas vinícolas com enoturismo, sendo os diretores ou gerentes de enoturismo os informanteschave. O questionário foi revisado por especialistas e, posteriormente, um préteste do questionário com 6 empresas foi realizado durante o mês de março de 2014, em outra área de enoturismo em Portugal, a região de Setúbal. Uma vez revisto e compreendido, o questionário final foi elaborado contendo perguntas de escala do tipo Likert, com 5 pontos, o qual examina as capacidades dinâmicas $(C D)$, o desenvolvimento do enoturismo (DE) e o desempenho organizacional (DO), como segue:

A capacidades dinâmicas (CD) capturam: (1) a geração, disseminação e capacidade de resposta às oportunidades de mercado por meio da capacidade de detectar (CDE), com 4 itens; (2) a aquisição, assimilação, transformação e exploração do conhecimento por meio da capacidade de aprender (CA), com 5 itens; (3) a contribuição, representação e inter-relação da contribuição individual de cada colaborador para a empresa por meio da capacidade de integrar (CI), com 5 itens; (4) a alocação de recursos, atribuição de tarefas e sincronização por meio da capacidade de coordenar (CC), com 5 itens; (5) e o potencial para a reconfiguração por meio da capacidade de reconfigurar (CR), com 2 itens. Todos estes itens foram adaptados a partir da escala proposta por Pavlou e El Sawy $(2011,2013)$, a qual foi validada no contexto da indústria eletrônica (Chen \& Chang, 2012) e no setor hoteleiro (Nieves \& Haller, 2014). 
O desenvolvimento do enoturismo (DE) é avaliado por um conjunto de atributos que compõem o produto enoturístico e que são identificados como relevantes pelos estudos experienciais sobre a demanda enoturística (Alant \& Bruwer, 2004; Alonso, 2005; Bruwer \& Lesschaeve, 2012; Bruwer, 2003; Carlsen \& Charters, 2006; Cohen \& Ben-Nun, 2009; Dowling \& Carlsen, 1998; Getz \& Brown, 2006; Hall et al., 2002; Mcdonnell \& Hall, 2008; Sparks, 2007; Stavrinoudis et al., 2012; Tassiopoulos, Nuntsu, \& Haydam, 2004; Zhang \& Qiu, 2011). Um total de 45 itens envolve aspectos físicos do interior e exterior da adega, recursos humanos, marketing, serviço ao cliente e atividades de enoturismo oferecidas nas vinícolas.

O desempenho organizacional (DO) mensura o impacto do desenvolvimento do enoturismo na organização por meio de 6 itens adaptados de Hung et al. (2007), que foram validados a partir de Hung et al. (2010).

O questionário foi disponibilizado on-line durante 10 semanas entre os meses de maio a agosto de 2014. A amostra para o estudo foi elaborada a partir de uma lista de 62 vinícolas com a componente de enoturismo na Rota dos Vinhos do Alentejo. Isto significa que as vinícolas pesquisadas têm, no mínimo, produção de vinho e recepção de visitantes para a visita e degustação de vinho nas suas instalações. A população-alvo foi convidada a participar da pesquisa voluntariamente, por telefone e e-mail. A partir dos questionários retornados, um total de 40 observações foram aceitas, o que representa uma alta taxa de resposta (64,5\%) em relação a outros estudos sobre desenvolvimento do enoturismo (Alonso \& Liu, 2012; Iglesias \& Navarro, 2014; Stavrinoudis et al., 2012).

\section{DADOS E MÉTODO DE ANÁLISE DOS DADOS}

O perfil das empresas da amostra $(n=40)$ consiste em vinícolas de pequeno à grande porte. A produção de vinho varia entre 5 mil a 18 milhões (litros/ano) e concentra-se em até 1 milhão de litros/ano para 75\% da amostra. A maioria das empresas (65\%) têm até 15 colaboradores permanentes e até 3 colaboradores envolvidos com as atividades de enoturismo, em que uma minoria da equipe de colaboradores tem qualificação em enoturismo. 
A pesquisa fez uso do pacote estatístico SPSS (versão 22) e do software SmartPLS -versão 3.1.5 (Ringle, Wende, \& Becker, 2014) para, respectivamente, descrever os dados e estimar o Modelo de Equações Estruturais (SEM). Verifica-se um uso cada vez maior da metodologia SEM em pesquisas na área do turismo (Nunkoo \& Ramkissoon, 2011; Pinto, 2016; Valle \& Assaker, 2015). Considerando o pequeno tamanho da amostra e a complexidade do modelo, a abordagem Partial Least Square Path Modeling (PLS-PM) foi considerada adequada para estimar e validar o modelo (Chin, 2010; Henseler, Ringle, \& Sinkovics, 2009; Valle \& Assaker, 2015). No que se refere aos requisitos mínimos para a dimensão da amostra, a "regra das dez vezes" (ten times rule) deve ser considerada para a aplicação da abordagem PLS (Barclay, Higgins \& Thompson, 1995). De acordo com esta regra, o tamanho da amostra deve exceder a relação mais complexa no modelo, isto é: a) dez vezes o número máximo de indicadores associados a uma variável latente com modelo de medida formativo; ou b) dez vezes o número máximo de caminhos que apontem para uma variável latente no modelo estrutural (Pinto, 2016). No modelo de análise desta pesquisa, os 40 casos obtidos representam cerca de 2/3 da população-alvo. Além disso, a regra das dez vezes é cumprida neste modelo.

A Figura 1 mostra o modelo seguindo a abordagem de Pavlou e El Sawy (2011, 2013), a qual propõe que a variável capacidades dinâmicas (CD) é um construto de segunda ordem com relações formativas com a capacidade de detectar (CDE), a capacidade de aprender (CA), capacidade de integrar (CI), a capacidade de coordenar (CC) e a capacidade de reconfigurar (CR). O modelo original de Pavlou e El Sawy $(2011,2013)$ posiciona a reconfiguração como uma medida interna do construto "capacidades dinâmicas", mas, no modelo de análise adotado nesta pesquisa, essa capacidade de reconfigurar é considerada uma quinta capacidade. Argumentos de David Teece e alguns estudos sustentam esta decisão (Fischer et al., 2010; Gebauer, 2011; Kindström et al., 2013).

Devido à relação formativa do construto CD com as 5 capacidades, o modelo pode ser classificado como um "construto molar de segunda ordem" (Chin, 2010) ou como um "modelo refletivo-formativo" (Becker, Klein, \& Wetzel, 2012), no sentido de que existe um "conceito geral" (que é o construto CD, no modelo da Figura 1), que é mediado pela influência dos construtos refletivos de primeira 
ordem (CDE, CA, CI, CC e CR), na variável endógena subsequente, desenvolvimento do enoturismo (DE). Assim, esta abordagem permite derivar o efeito indireto dos 5 construtos refletivos de primeira ordem (CDE, CA, CI, CC e CR) no DE como um produto de seus pesos para o construto formativo (CD) e o coeficiente de caminho que liga $C D$ e DE. Além desses construtos, o modelo também propõe que $D E$ e desempenho organizacional (DO) são construtos refletivos de primeira ordem.

Estudos apresentados por Becker et al. (2012) mostram que a abordagem de indicador repetido deve ser usada para os modelos refletivos-formativos e "esta abordagem produz geralmente menos enviesamento e, portanto, parâmetros estimados mais precisos e um score do construto de ordem superior mais confiável" (Becker et al., 2012, p. 376). Segundo esta abordagem, adotada no modelo de análise da Figura 1, o construto de segunda ordem é medido usando o mesmo conjunto de itens utilizados para medir cada construto de primeira ordem (Becker et al., 2012; Chin, Marcolin, \& Newsted, 2003; Chin, 2010). Adicionalmente, estudos empíricos com as capacidades dinâmicas também fornecem justificativas para a aceitação do modelo de segunda ordem (Protogerou et al., 2012; Wilden et al., 2013).

Após a estimação do modelo, partiu-se para a sua validação observando os resultados mais importantes para os dois componentes: o modelo de medida (refletivo e formativo) e o modelo estrutural (Hair, Ringle, \& Sarstedt, 2013; Henseler et al., 2009). Por fim, as hipóteses de pesquisa foram testadas por meio da observação do sinal e da significância estatística das relações diretas e indiretas entre os construtos latentes.

RESULTADOS

\section{MODELO DE MEDIDA}

No total, o modelo foi estimado com um tamanho de amostra de 40 casos, 8 variáveis latentes e 103 indicadores. A Tabela 1 apresenta os principais resultados do modelo de medida refletivo, permanecendo 55 indicadores. Os resultados do modelo de medida refletivo sugerem que os construtos utilizados neste 
estudo têm níveis satisfatórios de consistência interna (ou seja, fiabilidade), e validade (convergente e discriminante). Todos os itens possuem carga moderada à alta nos seus construtos latentes correspondentes (cargas excedem 0.592), sugerindo, pelo menos, moderada fiabilidade individual. A significância das cargas também é relatada na Tabela 1 (todos $p<0.01$ ). De notar que o modelo foi inicialmente estimado com mais itens, em especial no construto DE. No entanto, a fim de atender a todos os requisitos mínimos do modelo de medida em termos de fiabilidade e validade, estes itens precisaram ser eliminados. Este é um resultado esperado em função da natureza exploratória deste estudo.

Tabela 1: Modelo de medida refletivo

\begin{tabular}{|c|c|c|c|c|}
\hline Construtos & Indicadores & $\begin{array}{l}\text { Cargas } \\
\text { de } \\
\text { segunda } \\
\text { ordem }\end{array}$ & $\begin{array}{l}\text { Cargas } \\
\text { de } \\
\text { primeira } \\
\text { ordem }\end{array}$ & p-values \\
\hline \multirow{4}{*}{$\begin{array}{l}\text { Capacidade de } \\
\text { Detectar (CDE) }\end{array}$} & Analisar o mercado & 0.823 & 0.888 & \multirow{4}{*}{0.000} \\
\hline & Monitorizar efeitos de mudanças nos clientes & 0.695 & 0.846 & \\
\hline & Esforços de desenvolvimento de produtos & 0.803 & 0.911 & \\
\hline & Implementar ideias & 0.782 & 0.877 & \\
\hline \multirow{5}{*}{$\begin{array}{l}\text { Capacidade de } \\
\text { Aprender (CA) }\end{array}$} & Identificar novas informações & 0.828 & 0.890 & \multirow{5}{*}{0.000} \\
\hline & Assimilar novas informações & 0.799 & 0.905 & \\
\hline & $\begin{array}{c}\text { Transformar a informação em novo } \\
\text { conhecimento }\end{array}$ & 0.771 & 0.928 & \\
\hline & Usar o novo conhecimento & 0.744 & 0.923 & \\
\hline & Desenvolver novo conhecimento & 0.791 & 0.922 & \\
\hline \multirow{5}{*}{$\begin{array}{c}\text { Capacidade de } \\
\text { Integrar (CI) }\end{array}$} & Contribuição individual para o grupo & 0.593 & 0.748 & \multirow{5}{*}{0.000} \\
\hline & Entendimento global das tarefas de cada um & 0.800 & 0.913 & \\
\hline & Conhecimento e habilidades para as funções & 0.799 & 0.898 & \\
\hline & Interação entre departamentos & 0.605 & 0.738 & \\
\hline & Interligar atividades entre departamentos & 0.777 & 0.912 & \\
\hline \multirow{5}{*}{$\begin{array}{l}\text { Capacidade de } \\
\text { Coordenar (CC) }\end{array}$} & Sincronizar o trabalho & 0.761 & 0.831 & \multirow{5}{*}{0.000} \\
\hline & Alocar os recursos & 0.693 & 0.839 & \\
\hline & Atribuir tarefas & 0.645 & 0.732 & \\
\hline & Compatibilidade entre conhecimentos & 0.658 & 0.902 & \\
\hline & Coordenação da equipe & 0.642 & 0.795 & \\
\hline \multirow{2}{*}{$\begin{array}{c}\text { Capacidade de } \\
\text { Reconfigurar } \\
\text { (CR) }\end{array}$} & Reconfigurar recursos & 0.765 & 0.929 & \multirow{2}{*}{0.000} \\
\hline & Recombinar recursos & 0.794 & 0.935 & \\
\hline \multirow{7}{*}{$\begin{array}{l}\text { Desenvolvimento } \\
\text { do Enoturismo } \\
\text { (DE) }\end{array}$} & Recursos regionais & - & 0.666 & \multirow{7}{*}{0.000} \\
\hline & Formação em turismo & - & 0.772 & \\
\hline & Idioma de atendimento & - & 0.688 & \\
\hline & Familiaridade com processos & - & 0.707 & \\
\hline & Restaurante & - & 0.686 & \\
\hline & Atividades artísticas & - & 0.765 & \\
\hline & Atividades temáticas & - & 0.685 & \\
\hline
\end{tabular}




\begin{tabular}{cc}
\hline & Vantagem competitiva \\
Desempenho & Quota de mercado \\
Organizacional & Lucros \\
(DO) & Custos \\
& Vendas \\
& Satisfação dos clientes \\
\hline
\end{tabular}

Fonte: Elaboração própria.

A Tabela 2 apresenta os valores da variância média extraída (AVE), a fiabilidade do construto $(C R)$, e os valores de $R^{2}$ e $Q^{2}$ para as variáveis latentes endógenas. Para $C R$, os valores do modelo ultrapassam o limiar mínimo recomendado de 0.7 (Fornell \& Larcker, 1981). Além disso, todos os construtos revelam AVE superior a 0.5, sugerindo uma validade convergente adequada (Bagozzi \& Yi, 1988). Particularmente, isto significa que, em média, uma variável latente é capaz de explicar mais do que a metade da variância dos seus indicadores. Ao avaliar a validade discriminante, o critério de Fornell e Larcker (1981) e as cargas transversais foram observadas (valores não estão nas tabelas). Em relação ao anterior, observase que os valores de raiz quadrada de AVE de cada construto são maiores do que os valores do coeficiente de correlação de cada construto em relação aos outros construtos. No que respeita às cargas transversais, cada carga do indicador é maior no correspondente construto do que em outros construtos. Finalmente, os valores de $R^{2}$ medem a variabilidade explicada nas equações estruturais para $\mathrm{CDE}, \mathrm{CA}, \mathrm{CI}, \mathrm{CC}, \mathrm{DE}$ e DO. E os valores de $\mathrm{Q}^{2}$ são todos positivos, o que significa que as equações estruturais correspondentes têm relevância preditiva.

Tabela 2: Avaliação do modelo de medida

$\begin{array}{ccccc} & \text { AVE } & \text { CR } & \mathbf{R}^{\mathbf{2}} & \mathbf{Q}^{\mathbf{2}} \\ \text { Capacidades Dinâmicas (CD)* } & - & 0.963 & 1.000 & 0.538 \\ \text { Capacidade de Detectar (CDE) } & 0.776 & 0.933 & - & - \\ \text { Capacidade de Aprender (CA) } & 0.835 & 0.962 & - & - \\ \text { Capacidade de Integrar (CI) } & 0.715 & 0.926 & - & - \\ \text { Capacidade de Coordenar (CC) } & 0.675 & 0.912 & - & - \\ \text { Capacidade de Reconfigurar (CR) } & 0.869 & 0.930 & - & - \\ \text { Desenvolvimento do Enoturismo (DE) } & 0.506 & 0.877 & 0.529 & 0.224 \\ \text { Desempenho Organizacional (DO) } & 0.643 & 0.914 & 0.394 & 0.222 \\ \text { *: Construto de segunda ordem. } & & & & \end{array}$

Fonte: Elaboração própria.

Finalmente, na avaliação do construto de segunda ordem (CD), observase que os cinco pesos são estatisticamente significativos, o que significa que 
a capacidade de detectar $(\mathrm{CDE})$ (peso $=0.256 ; \mathrm{p}=0.000$ ), a capacidade de aprender $(C A)$ (peso $=0.295 ; p=0.000)$, a capacidade de integrar $(\mathrm{CI})$ (peso $=0.263 ; p=0.000)$, a capacidade de coordenar $(C C)($ peso $=0.233 ; p=0.000$ ) e a capacidade de reconfigurar $(C R)$ (peso $=0.121 ; p=0.000$ ) contribuem de forma significativa para formar o construto $C D$. Além disso, os valores do fator de inflação da variância (VIF), nas cinco situações são muito baixos, claramente inferiores a 5, o que revela ausência de séria multicolinearidade em um conjunto de indicadores relacionados ao construto formativo (isto é, CD neste modelo de análise).

\section{MODELO ESTRUTURAL}

As estimativas do coeficiente de caminho são apresentadas na Tabela 3. Estes são os coeficientes estimados para as relações diretas e indiretas, no que diz respeito a $\mathrm{H} 1$ ( $a, b, c, d, e)$, e a H2. Estes resultados e os correspondentes valores de $p$-values são resultados do software SmartPLS. Nenhuma hipótese é rejeitada nesta pesquisa ( $p<0.05)$.

Tabela 3: Efeitos diretos e indiretos e hipóteses de investigação

Hipóteses

$\mathrm{H} 1$

$\mathrm{H} 1 \mathrm{a}$

$\mathrm{H} 1 \mathrm{~b}$

$\mathrm{H} 1 \mathrm{C}$

$\mathrm{H} 1 \mathrm{~d}$

$\mathrm{H} 1 \mathrm{e}$

$\mathrm{H} 2$

\section{Relações entre construtos}

$C D>D E$

$\mathrm{CDE}>\mathrm{DE}^{*}$

$C A>D^{*}$

$\mathrm{CI}>\mathrm{DE}^{*}$

$C C>D E^{*}$

$\mathrm{CR}>\mathrm{DE}^{*}$

$\mathrm{DE}>\mathrm{DO}$

\section{Coeficientes $\boldsymbol{\beta}$}

0.727

0.186

0.214

0.191

0.170

0.088

0.628

\section{p-value (bootstrap)}

0.000

0.000

0.000

0.000

0.000

0.000

0.000

\section{Verificação das \\ hipóteses}

Não rejeitada

Não rejeitada

Não rejeitada

Não rejeitada

Não rejeitada

Não rejeitada

Não rejeitada

*: Efeitos indiretos.

Fonte: Elaboração própria.

Os efeitos totais das variáveis latentes exógenas sobre o DE e o DO são representados na Tabela 4. Como pode ser visto, a variável capacidade de aprender (CA) tem maior incidência no desenvolvimento do enoturismo (efeito total $=0.214$ ) e no desempenho organizacional $(D O)$ (efeito total $=0.120$ ). Todos os efeitos totais são estatisticamente significativos.

Tabela 4: Análise dos efeitos totais das variáveis independentes no desenvolvimento do enoturismo 


$\begin{array}{lccc} & & \beta & \text { p-value (bootstrap) } \\ C D E & D E & 0.186 & 0.000 \\ C A & D E & 0.214 & 0.000 \\ C I & D E & 0.191 & 0.000 \\ C C & D E & 0.170 & 0.000 \\ C R & D E & 0.088 & 0.000 \\ C D E & D O & 0.117 & 0.000 \\ C A & D O & 0.134 & 0.000 \\ C I & D O & 0.120 & 0.000 \\ C C & D O & 0.106 & 0.000 \\ C R & D O & 0.055 & 0.000\end{array}$

Fonte: Elaboração própria.

A análise dos efeitos indiretos permite identificar as capacidades que têm maior incidência no DE nas vinícolas alentejanas. As capacidades de aprender, integrar e detectar são as mais fortes, seguidas pelas capacidades de coordenar e reconfigurar (Tabelas 3 e 4).

A capacidade de aprender é a habilidade de renovar as capacidades operacionais existentes com novos conhecimentos (Pavlou \& El Sawy, 2011, 2013). Para desenvolver o enoturismo, é necessário que as vinícolas criem novos espaços na propriedade agrícola para receber os visitantes na adega e ofereçam atividades turísticas combinadas com o vinho. Esta expansão no modelo de negócio envolve um processo de aprendizagem interno, por meio de rotinas organizacionais específicas, tais como: (a) adquirir novas informações e conhecimentos, (b) assimilar estas novas informações e conhecimentos, (c) transformar a informação existente em novo conhecimento sobre enoturismo, (d) utilizar o conhecimento em novas atividades/serviços para o enoturismo, e (e) desenvolver novos conhecimentos que têm o potencial de influenciar o desenvolvimento de atividades de enoturismo na unidade de negócio da empresa.

A capacidade de integrar está relacionada com a lógica coletiva e com os padrões de interação compartilhados (Pavlou \& El Sawy, 2011, 2013). No contexto das vinícolas alentejanas, as atividades de enoturismo devem contribuir com conhecimento e informação para outros departamentos da empresa, como os setores voltados para a produção vinícola, de vendas e marketing.

A capacidade de detectar é a habilidade organizacional para examinar o ambiente, a fim de identificar oportunidades de mercado e necessidades dos 
clientes (Teece et al., 1997). O enoturismo se encaixa em uma nova oportunidade de mercado para as vinícolas e vai ao encontro das necessidades dos clientes atuais que procuram conhecer e aprender sobre vinhos, desfrutar dos ambientes rurais e da gastronomia local.

A capacidade de coordenar requer a sincronização do enoturismo com outras atividades e departamentos, fazendo com que as visitas e as atividades de enoturismo não interfiram (ao ponto de atrapalhar) nas atividades agrícolas diárias de produção de vinho. E, por fim, a capacidade de reconfigurar envolve rotinas para reconfigurar os recursos que levam a novos ativos produtivos. Estas empresas devem, frequentemente, se envolver na recombinação de recursos por meio da implementação de novos métodos de gestão para o enoturismo e de procedimentos de alocação de recursos humanos para melhor corresponder às necessidades dos visitantes e aproveitar os seus ativos organizacionais.

\section{CONSIDERAÇÕES FINAIS}

O referencial teórico das capacidades dinâmicas permite identificar que as vinícolas alentejanas constroem e utilizam capacidades dinâmicas que as permitem reconfigurar recursos e habilidades em novas capacidades em direção ao DE. Assim, a relação direta e positiva das capacidades dinâmicas com o DE é suportada $(\mathrm{H} 1)$.

Este trabalho identificou que um conjunto de capacidades - detectar, aprender, coordenar, integrar e reconfigurar - contribui simultaneamente e de forma diferente para o $\mathrm{DE}(\mathrm{H} 1 \mathrm{a}, \mathrm{b}, \mathrm{c}, \mathrm{d}, \mathrm{e})$. Este resultado é consistente com outras pesquisas (Fischer et al., 2010; Gebauer, 2011; Kindström et al., 2013; Pavlou \& El Sawy, 2011, 2013).

Em relação ao segundo objetivo desta pesquisa, os resultados mostram que o DE promove um melhor desempenho para as vinícolas $(\mathrm{H} 2)$, em termos de vantagem competitiva, quota de mercado, lucros, vendas e satisfação do cliente, enquanto alguns custos com produtos e serviços podem ser reduzidos (Tabela 1). Resultados semelhantes são identificados em pequenas vinícolas na Nova Zelândia (Beverland \& Lockshin, 2001). 
A partir dessa perspectiva intraorganizacional identificada e definida, este estudo revela como as vinícolas mudam processos internos utilizando capacidades dinâmicas que permitam a elas se envolver com a indústria do turismo por meio do enoturismo. Este resultado mostra que as vinícolas renovam e ampliam as suas capacidades operacionais.

\section{IMPLICAÇÕES, RECOMENDAÇÕES E LIMITAÇÕES}

A contribuição deste estudo envolve, principalmente, três aspectos: a aplicação das capacidades dinâmicas, a metodologia de Modelação de Equações Estruturais (SEM) e a perspectiva das vinícolas numa pesquisa sobre o DE. Assim, este trabalho contribui para a área de conhecimento em termos de testar teoria e fornecer uma série de indicadores válidos e testados, estimulando pesquisas futuras. Especificamente, este trabalho preenche uma lacuna de investigação empírica sobre enoturismo por meio da aplicação da abordagem de capacidades dinâmicas. Existe uma grande quantidade de estudos teóricos sobre as capacidades dinâmicas, mas ainda há espaço para estudos quantitativos. Este é um dos aspectos inovadores deste estudo. A outra inovação é a aplicação da metodologia SEM na perspectiva da oferta, uma vez que ela é geralmente utilizada na perspectiva da demanda em estudos sobre atitudes e comportamentos turísticos.

Esta pesquisa incorpora cinco capacidades dinâmicas que conduzem o processo de inovação em direção ao enoturismo, mostrando que as vinícolas renovam e ampliam as suas capacidades operacionais. Além disso, elas refletem sobre como o modelo de negócio pode ser reconfigurado (com a agregação do enoturismo), principalmente quando o ambiente no qual a empresa está inserida tem características instáveis e é altamente competitivo.

Este trabalho apresenta uma série de implicações práticas para a realidade empresarial das indústrias do vinho e do turismo, no que diz respeito à natureza e à gestão do enoturismo. Do ponto de vista gerencial, este artigo fornece orientações sobre a importância de investir em capacidades dinâmicas e em como elas podem ser aproveitadas na empresa. Organizações em ambientes 
altamente competitivos (como o setor do turismo) devem ser guiadas por gestores que precisam ser empreendedores e inovadores. Assim, a criação e a implementação de capacidades dinâmicas (detectar, aprender, integrar, coordenar e reconfigurar) são essenciais. As capacidades dinâmicas também facilitam o foco da vinícola para a expansão do modelo de negócio ao envolver atividades ligadas ao serviço turístico por meio do enoturismo. Em geral, estes resultados são particularmente interessantes para os processos de decisão gerencial que almejam o sucesso organizacional e as vantagens competitivas.

A principal limitação deste estudo é não permitir a generalização dos resultados, pois é baseado em uma amostra e em um território específico. Futuros trabalhos podem resolver esta limitação com a replicação em outras regiões de enoturismo.

\section{REFERÊNCIAS}

Alant, K., \& Bruwer, J. (2004). Wine tourism behaviour in the context of a motivational framework for wine regions and cellar doors. Journal of Wine Research, 15(1), 27-37.

Alonso, A. D. (2005). Wine tourism experiences in New Zealand: An exploratory study. Tese de doutorado não publicada. Lincoln University, Nova Zelândia.

Alonso, A. D., \& Liu, Y. (2012). Old wine region, new concept and sustainable development: Winery entrepreneurs' perceived benefits from wine tourism on Spain's Canary Islands. Journal of Sustainable Tourism, 20(7), 991-1009.

Ambrosini, V., \& Bowman, C. (2009). What are dynamic capabilities and are they a useful construct in strategic management? International Journal of Management Reviews, 11(1), $29-49$.

Ambrosini, V., Bowman, C., \& Collier, N. (2009). Dynamic capabilities: An exploration of how firms renew their resources base. British Journal of Management, 20(S1), S9-S24.

Bagozzi, R. P., \& Yi, Y. (1988). On the evaluation of structural equation models. Journal of the Academy of Marketing Science, 16(1), 74-94.

Barclay, D. W., Higgins, C. A. \& Thompson, R. (1995). The partial least squares approach to causal modeling: Personal computer adoption and use as illustration. Technology Studies, 2(2), 285-309.

Barrales-Molina, V., Martínez-López, F. J., \& Gázquez-Abad, J. C. (2014). Dynamic marketing capabilities: Toward an integrative framework. International Journal of Management Reviews, 16, 397-416. 
Barreto, I. (2010). Dynamic capabilities: A review of past research and an agenda for the future. Journal of Management, 36(1), 256-280.

Beames, G. (2003). The rock, the reef and the grape: The challenges of developing wine tourism in regional Australia. Journal of Vacation Marketing, 9(3), 205-212.

Becker, J.-M., Klein, K., \& Wetzels, M. (2012). Hierarchical latent variable models in PLS-SEM: Guidelines for using reflective-formative type models. Long Range Planning, 45(5-6), 359-394.

Beverland, M., \& Lockshin, L. S. (2001). Organizational life cycles in small New Zealand wineries. Journal of Small Business Management, 39(4), 354-362.

Bruwer, J. (2003). South African wine routes: Some perspectives on the wine tourism industry's structural dimensions and wine tourism product. Tourism Management, 24(4), 423-435.

Bruwer, J., \& Lesschaeve, I. (2012). Wine tourists' destination region brand image perception and antecedents: Conceptualization of a winescape framework. Journal of Travel \& Tourism Marketing, 29(7), 611-628.

Carlsen, J. (2004). A review of global wine tourism research. Journal of Wine Research, 15(1), 5-13.

Carlsen, J., \& Charters, S. (2006). Global wine tourism: Research, management and marketing. London: $C A B$ International.

Chen, Y.-S., \& Chang, C.-H. (2012). The determinants of green product development performance: Green dynamic capabilities, green transformational leadership, and green creativity. Journal of Business Ethics, 116(1), 107-119.

Chin, W. (2010). How to write up and report PLS analyses. In V. E. Vinzi, W. W. Chin, J. Henseler, \& H. Wang (Eds.), Handbook of Partial Least Squares (pp. 655-689). Berlin: Springer Handbooks of Computational Statistics.

Chin, W. W., Marcolin, B. L., \& Newsted, P. R. (2003). A partial least squares latent variable modeling approach for measuring interaction effects: Results from a Monte Carlo simulation study and an electronic-mail emotion/adoption study. Information Systems Research, 14(2), 189-217.

Christou, E., \& Nella, A. (2010). A review of wine tourism research from 1995 to 2010: Analysis of 110 contributions. Journal of Hospitality and Tourism, 8, 2-15.

Cohen, E., \& Ben-Nun, L. (2009). The important dimensions of wine tourism experience from potential visitors' perception. Tourism and Hospitality Research, 9(1), 20-31.

Desai, D., Sahu, S., \& Sinha, P. K. (2007). Role of dynamic capability and information technology in customer relationship management: A study of Indian companies. VIKALPA, 32(4), 45-62. 
Dowling, R., \& Carlsen, J. (1998). Wine tourism: Perfect partners. Proceedings of the First Australian Wine Tourism Conference. Margaret River, Australia: Edith Cowan University.

Easterby-Smith, M., Lyles, M. A., \& Peteraf, M. A. (2009). Dynamic capabilities: Current debates and future directions. British Journal of Management, 20, S1-S8.

Eisenhardt, K. M., \& Martin, J. A. (2000). Dynamic capabilities: What are they? Strategic Management Journal, 21(10-11), 1105-1121.

Ellonen, H.-K., Wikström, P., \& Jantunen, A. (2009). Linking dynamic capability portfolios and innovation outcomes. Technovation, 29(11), 753-762.

Fischer, T., Gebauer, H., Gregory, M., Ren, G., \& Fleisch, E. (2010). Exploitation or exploration in service business development?: Insights from a dynamic capabilities perspective. Journal of Service Management, 21(5), 591-624.

Fornell, C., \& Larcker, D. F. (1981). Evaluating structural equation models with unobservable variables and measurement error. Journal of Marketing Research XVIII, 39-50.

García-Morales, V. J., Jiménez-Barrionuevo, M. M., \& Gutiérrez-Gutiérrez, L. (2012). Transformational leadership influence on organizational performance through organizational learning and innovation. Journal of Business Research, 65(7), 1040-1050.

Gebauer, H. (2011). Exploring the contribution of management innovation to the evolution of dynamic capabilities. Industrial Marketing Management, 40(8), 1238-1250.

Getz, D. (2000). Explore wine tourism: Management, development and destinations. New York: Cognizant Communication Corporation.

Getz, D., \& Brown, G. (2006). Critical success factors for wine tourism regions: A demand analysis. Tourism Management, 27(1), 146-158.

Grande, J. (2011). New venture creation in the farm sector: Critical resources and capabilities. Journal of Rural Studies, 27(2), 220-233.

Hair, J. F., Ringle, C. M., \& Sarstedt, M. (2013). Partial least squares structural equation modeling: Rigorous applications, better results and higher acceptance. Long Range Planning, 46(1-2), 1-12.

Hall, C. M., Sharples, L., Cambourne, B., \& Macionis, N. (2002). Wine tourism around the world. Oxford: Butterworth-Heinemann.

Haugland, S. A., Ness, H., Grønseth, B.-O., \& Aarstad, J. (2011). Development of tourism destinations: An integrated multilevel perspective. Annals of Tourism Research, 38(1), 268-290.

Helfat, C. E., Finkelstein, S., Mitchell, W., Peteraf, M. A., Singh, H., Teece, D. J., \& Winter, S. (2007). Dynamic capabilities: Understanding strategic change in organizations. Malden, MA: Blackwell Publishing. 
Henseler, J., Ringle, C. M., \& Sinkovics, R. R. (2009). The use of partial least squares path modeling in international marketing. Advances in International Marketing, 20, 277-319.

Hertog, P. den, Aa, W. Van der, \& Jong, M. W. de. (2010). Capabilities for managing service innovation: Towards a conceptual framework. Journal of Service Management, 21(4), 490-514.

Hojman, D. E., \& Hunter-Jones, P. (2012). Wine tourism: Chilean wine regions and routes. Journal of Business Research, 65(1), 13-21.

Hsu, L.-C., \& Wang, C.-H. (2012). Clarifying the effect of intellectual capital on performance: The mediating role of dynamic capability. British Journal of Management, 23(2), 179-205.

Hung, R. Y.-Y., Chung, T., \& Lien, B. Y.-H. (2007). Organizational process alignment and dynamic capabilities in high-tech industry. Total Quality Management \& Business Excellence, 18(9), 1023-1034.

Hung, R., Yang, B., Lien, B. Y.-H., McLean, G. N., \& Kuo, Y.-M. (2010). Dynamic capability: Impact of process alignment and organizational learning culture on performance. Journal of World Business, 45(3), 285-294.

Iglesias, M. P., \& Navarro, M. M. (2014). Desarrolo del enoturismo desde la perspectiva de las bodegas familiares. Cuadernos de Turismo, 34, 233-249.

Instituto da Vinha e do Vinho, I. P. (2015). Wine sector statistics in the year 2013. Recuperado em 2 fevereiro, 2015, de http://www.ivv.min-agricultura.pt/

Kim, J., \& Boo, S. (2010). Dynamic Capabilities and Performance of Meeting Planners. Journal of Travel \& Tourism Marketing, 27(7), 736-747.

Kindström, D., Kowalkowski, C., \& Sandberg, E. (2013). Enabling service innovation: A dynamic capabilities approach. Journal of Business Research, 66(8), 1063-1073.

Lavandoski, J., Vargas-Sánchez, A., \& Silva, J. A. (2014). Significado e importância da abordagem de capacidades dinâmicas nos estudos turísticos: Uma revisão de literatura. In M. Santos, P. Águas, F. Serra, \& J. A. C. Santos (Eds.), TMS Conference Series - Perspectivas Contemporâneas em Turismo (pp. 317-330). Faro: Universidade do Algarve, Escola Superior de Gestão, Hotelaria e Turismo.

Lemmetyinen, A., \& Go, F. M. (2009). The key capabilities required for managing tourism business networks. Tourism Management, 30(1), 31-40.

Leonidou, L. C., Leonidou, C. N., Fotiadis, T. A., \& Zeriti, A. (2013). Resources and capabilities as drivers of hotel environmental marketing strategy: Implications for competitive advantage and performance. Tourism Management, 35, 94-110. 
Macionis, N. (1997). Wine tourism in Australia: Emergence, development and critical issues. Tese de doutorado não publicada. University of Canberra, Australia.

McDonnell, A., \& Hall, C. M. (2008). A framework for the evaluation of winery servicescapes: A New Zealand case. Pasos Revista de Turismo Y Patrimonio Cultural, 6(2), 231-247.

Nieves, J., \& Haller, S. (2014). Building dynamic capabilities through knowledge resources. Tourism Management, 40, 224-232.

Nunkoo, R., \& Ramkissoon, H. (2011). Structural equation modelling and regression analysis in tourism research. Current Issues in Tourism, 15(8), 777-802.

Pavlou, P. A., \& El Sawy, O. A. (2011). Understanding the elusive black box of dynamic capabilities. Decision Sciences, 42(1), 239-273.

Pavlou, P. A., \& El Sawy, O. A. (2013). Searching for a simple model of dynamic capabilities. Social Science Electronic Publishing, 1-35. Recuperado de http://papers.ssrn.com/sol3/ papers.cfm?abstract_id $=2369378$

Pinto, Patrícia. (2016). Modelos de equações estruturais com variáveis latentes: Fundamentos da abordagem Partial Least Squares. Bnomics.

Prahalad, C. K., \& Hamel, G. (1990). The core competence of the corporation'. Harvard Business Review, 68(3), 79-91.

Protogerou, A., Caloghirou, Y., \& Lioukas, S. (2012). Dynamic capabilities and their indirect impact on firm performance. Industrial and Corporate Change, 21(3), 615-647.

Ringle, C. M., Wende, S., \& Becker, J.-M. (2014). Smartpls3 (versão 3.1.5). [Software]. Hamburg: SmartPLS. Recuperado de www.smartpls.com

Sainaghi, R., \& De Carlo, M. (2012). Developing dynamic capabilities in a tourism destination: An organizational approach. Proceedings of the 12 Euram. Rotterdam, Olanda: Rotterdam School of Management.

Salunke, S., Weerawardena, J., \& McColl-Kennedy, J. R. (2011). Towards a model of dynamic capabilities in innovation-based competitive strategy: Insights from project-oriented service firms. Industrial Marketing Management, 40(8), 1251-1263.

Sparks, B. (2007). Planning a wine tourism vacation? Factors that help to predict tourist behavioural intentions. Tourism Management, 28(5), 1180-1192.

Stavrinoudis, T. A., Tsartas, P., \& Chatzidakis, G. (2012). Study of the major supply factors and business choices affecting the growth rate of wine tourism in Greece. Current Issues in Tourism, 15(7), 627-647. 
Tassiopoulos, D., Nuntsu, N., \& Haydam, N. (2004). Wine tourists in South Africa: A demographic and psychographic study. Journal of Wine Research, 15(1), 51-63.

Teece, D. J. (2007). Explicating dynamic capabilities: The nature and microfoundations of (sustainable) enterprise performance. Strategic Management Journal, 28, 1319-1350.

Teece, D. J. (2009). Dynamic capabilities and strategic management. Oxford: Oxford University Press.

Teece, D., \& Pisano, G. (1994). The dynamic capabilities of firms: An introduction (Working Paper No. 94-103). Laxenburg, Austria: International Institute for Applied Systems Analysis.

Teece, D., Pisano, G., \& Shuen, A. (1997). Dynamic capabilities and strategic management. Strategic Management Journal, 18(7), 509-533.

Telfer, D. J. (2001). Strategic alliances along the Niagara wine route. Tourism Management, $22,21-30$.

Turismo de Portugal. (2013). Plano Estratégico Nacional do Turismo: revisão e objetivos 20132015. Lisboa: Turismo de Portugal, IP. Recuperado de http://www.turismodeportugal.pt/

Valle, P., \& Assaker, G. (2015). Using partial least squares structural equation modeling in tourism research: A review of past research and recommendations for future applications. Journal of Travel Research, 1-14.

Wilden, R., Gudergan, S. P., Nielsen, B. B., \& Lings, I. (2013). Dynamic capabilities and performance: Strategy, structure and environment. Long Range Planning, 46(1-2), 72-96.

Winter, S. G. (2003). Understanding dynamic capabilities. Strategic Management Journal, 24(10), 991-995.

Yalcinkaya, G., Calantone, R. J., \& Griffith, D. A. (2007). An examination of exploration and exploitation capabilities: Implications for product innovation and market performance. Journal of International Marketing, 15(4), 63-93.

Yien, J.-M., Chen, K.-H., Huang, K.-P., \& Huang, C.-J. (2011). Managerial decision and resource reallocation: A dynamic capability perspective. Jounal of Social Sciences, 7(4), 632-634.

Zahra, S. A., \& George, G. (2002). Absorptive capacity: A review, reconceptualization, and extension. Academy of Management Journal, 27(2), 185-203.

Zahra, S. A., Sapienza, H. J., \& Davidsson, P. (2006). Entrepreneurship and dynamic capabilities: A review, model and research agenda. Journal of Management Studies, 43(4), 917-955.

Zamora, J., \& Barril, M. E. (2007). Turismo y vino: Un estudio formativo sobre la evolución de las rutas del vino en Chile. Estudios Y Perspectivas En Turismo, 16, 173-194. 
Zhang, X., \& Qiu, C. (2011). Research on the development of wine tourism product based on the analysis of the wine tourist behavioral intentions: The case of Dynasty winery. Proccedings of the 2nd International Conference on Artificial Intelligence, Management Science and Electronic Commerce (pp. 1439-1442). Deng Leng, China: IEEE.

Zollo, M., \& Winter, S. G. (2002). Deliberate learning and the evolution of dynamic capabilities. Organization Science, 13(3), 339-351.

\section{CONTRIBUIÇÃO DOS AUTORES NA CONSTRUÇÃO DO ARTIGO}

LAVANDOSKI: 40\% - Introdução, revisão teórica, metodologia, resultados.

SILVA: 20\% - Metodologia e conclusão.

VARGAS-SÁNCHEZ: 20\% - Metodologia e conclusão.

PINTO: 20\% - Metodologia e conclusão. 